\title{
Design of Human Adaptive Mechatronics Controller for Upper Limb Motion Intention Prediction
}

\author{
R. Joshua Samuel Raj ${ }^{1, *}$, J. Prince Antony Joel ${ }^{2}$, Salem Alelyani ${ }^{3}$, Mohammed Saleh Alsaqer ${ }^{3}$ and \\ C. Anand Deva Durai
}

\footnotetext{
${ }^{1}$ Department of Information Science \& Engineering, CMR Institute of Technology, Bengaluru, India

${ }^{2}$ Department of Mechatronics Engineering, Rajas Engineering College, Tirunelveli, India

${ }^{3}$ Center for Artificial Intelligence, King Khalid University, Abha, Kingdom of Saudi Arabia

${ }^{4}$ College of Computer Science, King Khalid University, Abha, Kingdom of Saudi Arabia

*Corresponding Author: R. Joshua Samuel Raj. Email: joshuasamuelraj@gmail.com

Received: 09 July 2021; Accepted: 17 August 2021
}

\begin{abstract}
Human Adaptive Mechatronics (HAM) includes human and computer system in a closed loop. Elderly person with disabilities, normally carry out their daily routines with some assistance to move their limbs. With the short fall of human care takers, mechatronics devices are used with the likes of exoskeleton and exosuits to assist them. The rehabilitation and occupational therapy equipments utilize the electromyography (EMG) signals to measure the muscle activity potential. This paper focuses on optimizing the HAM model in prediction of intended motion of upper limb with high accuracy and to increase the response time of the system. Limb characteristics extraction from EMG signal and prediction of optimal controller parameters are modeled. Time and frequency based approach of EMG signal are considered for feature extraction. The models used for estimating motion and muscle parameters from EMG signal for carrying out limb movement predictions are validated. Based on the extracted features, optimal parameters are selected by Modified Lion Optimization (MLO) for controlling the HAM system. Finally, supervised machine learning makes predictions at different points in time for individual sensing using Support Vector Neural Network (SVNN). This model is also evaluated based on optimal parameters of motion estimation and the accuracy level along with different optimization models for various upper limb movements. The proposed model of human adaptive controller predicts the limb movement by $96 \%$ accuracy.
\end{abstract}

Keywords: Exoskeleton; electromyography (emg); human adaptive mechatronics; occupational therapy; motion prediction; machine learning

\section{Introduction}

Ageing brings upon health related costs and difficulty in locomotion is the most common thing affecting the elderly persons. Wearable orthotic devices can possibly develop the quality of

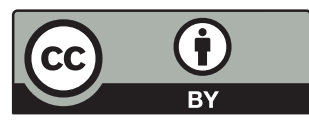

This work is licensed under a Creative Commons Attribution 4.0 International License, which permits unrestricted use, distribution, and reproduction in any medium, provided the original work is properly cited. 
daily activities for some elderly and disabled persons by preventing damage, restoring capacity, and expanding human physical limits [1] making them live more independently. Shortfall of caretakers and nursing assistance is predicted in near future. A computerized system could be fitting for exactness and dull undertakings in organized situations which still cannot be reasonable for certain assignments in amorphous conditions that demand abilities of quick assessment, flexibility and adjustment [2]. EMG signals are bio-electric signals that measure electric potential generated within the cell body to identify the neuromuscular activity. EMG signals have a wide range of uses, as applicable in restoration and assistive advancements, ergonomics, clinical analysis and game science [3]. Numerous technologies can be utilized to make a prediction of HAM movements. Human Adaptive mechatronics includes a keen mechatronics framework which adjusts to the skills of humans, in different situations for improving human abilities. Adaptive tech is characterized as an object, which is explicitly intended to build/keep up the capacities of individuals with inabilities. Gait disabilities mainly prevent individuals from being able to perform definite options to play out certain essential functions on their hold like Sitting and standing, strolling, getting a handle on and lifting an object. The inability to play out these functions does not just influence the autonomy of people with incapacities but also may meddle with their family.

Adaptive innovation is a sort of assistive tech. where, alteration of existing devices is adjusted for the utilization by individuals with inabilities. A large number of individuals around the globe need a HAM device every day to upgrade their living. When picking among different articles, a person looks bit by bit and moves toward the end for chosen item [4], furthermore, the last gaze fixation goes before the beginning of the arriving motion [5]. Two properties of HAM: Exoskeleton wearer expertise level like elements and movements can be assessed to improve the wearer ability. On the off chance that a help control of an incredible activity is conceivable, the wearer's capacity is improved from the first level. HAM requires the comprehension of humans. At the point when an individual is proposed to one physical action, the mind controls the musculoskeletal framework through nerves. The EMG-based limb movement prediction can be extensively ordered as characterization and relapse models. Discrete motion control from the collected EMG signals draws in numerous analysts [6]. Optimization of human ergonomics of the creation system starts with the predictor testing with prepared and untrained information. Accordingly, the general precision of expectation is brilliant with EMG levels listed on the flexors motion of the finger with respect to the wrist position in the flexion-augmentation and ulnar-radial plan [7].

In modern exoskeleton controllers, electronics components and sensors including the battery are bound inside [8]. Exoskeleton mounted over the body of the patient and joints can be legitimately adjusted with Servo motors which are widely used as the actuators and are same as the rehabilitation framework mechanisms $[9,10]$. The greater energy source adds additional load to the exoskeleton plan. To construct a vitality proficient component, further research is required on vitality optimization methods Genetic Algorithm (GA), Particle Swarm Optimization (PSO), Cuckoo Search (CS) [11,12]. In any case, human motor control may think on large assignment qualities as opposed to optimizing at least one of the features [13]. The least difficulty is in the artificial neural system models that acknowledge EMG signals as a contribution to predicting the position/velocity of a gadget. In any case, the anticipated development directions are not accurate [14]. To approve the exhibition of deep learning, three foreseeing components, specifically, SVM, MNLR, NN, etc., were applied to anticipate the HAM power from the EMG signals of forearm muscles in disconnected mode [15]. This encounter decreases the amplitude of the deliberate sign and along these lines influences the exactness of intended motion. Electrodes used for collecting EMG signals may shift or loose contact due to high resistance of the skin which may cause signal failure. It is a difficult assignment, and different techniques have been proposed to address this issue, such as human development modeling [16]. 
The major contribution of the work is to predict the limb movement using EMG signals with novel techniques. EMG signals are bio-electric signals that measure electric potential generated within the cell body to identify the neuromuscular activity and have a wide scope of utilizations. Initially, the features are extracted from EMG signals and the optimal features are selected using optimization techniques. The result of the optimal model is proposed movement, in view of acceleration; speed and torque which are analyzed by SVNN indicator.

\section{Summary of Literatures}

The utilization of Recurrent Neural Systems (RNNs) to develop the online characterization of HAM motions with EMG signals are obtained from upper limb muscles [17], and it was shown that the Feed Forward Neural Network (FFNN) and Long Short Term Memory (LSTM), RNN, and Gated Recurrent Units (GRU) accomplished comparable accuracy for the two informational collections. Dataset comprising of the EMG signals were gathered from 11 subjects at five diverse upper appendage positions. The time-space control spectral descriptors are utilized as the list of capabilities to prepare the DNN classifier. As opposed to the earlier strategies, the proposed methodology bars the component dimensionality to decrease steps that fundamentally diminish the general unpredictability [18]. A hand gesture acknowledgment model which reacts rapidly with qualified great exactness, is a reflexive technique for feature extraction from the time arrangement of shifting length, to long last the code. The dataset utilized for this work, is made openly accessible by Benalcazar et al. [19]. Surface Electromyography (SEMG) signals established an extensive function in various fields containing sports medicine, rehabilitation medicine, and clinical diagnosis. Convolution Neural Network (CNN')s exhibited great interpretation invariance because of its qualities of nearby association and weight sharing [20]. On the off chance that SEMG signals were functional in the representative of electromyography signal wave, the assorted variety of electromyography signal itself can be beaten utilizing invariance in convolutions. EMG signal was given to the versatile channel to yield the ideal loads utilizing the eminent optimization algorithms, known as firefly algorithm and Levenberg Marquardt (FLM). These algorithms are viably hybridized and functional to the neural system to locate the ideal loads for versatile sifting [21]. FLM optimization-based learning algorithm for neural system upgraded adaptive separating model which is acquainted with wiping out relics from the EMG.

\section{System Model}

The EMG-based expectation forecast of HAM movement can be comprehensively arranged as order and regression models. In numerous occasions authors have analyzed the EMG signals by means of supervised and unsupervised learning models. In our creative research work, motion is analyzed by model-based controller approach, where four stages are considered for the EMG examination which is (i) Preprocessing of EMG signals (ii) Selection of features by time and frequency-based method's (iii) Motion estimation through MLO model (iv) HAM motion estimated by SVNN.

\subsection{Robotic System}

Robot motion investigation is significant in every single development. The motivations behind this editorial exoskeletons and orthoses are characterized as mechanical gadgets that are basically human in nature imitations. Exoskeletons are wearable and fit near the body and work together with the user for motion enhancement [22]. Humans utilize three essential muscles to produce developments close to wrists, which are Flexor Digitorum Superficialis (A1), Flexor Carpi Radialis (A2), and Extensor 
Carpi Radialis Longus muscles (A3). A detailed clarity of this development is depicted in the following section.

A1: Its muscle is the biggest muscle in the forearm. It shapes a middle of the road muscle layer between the shallow and deep muscle gatherings. The median nerve and the ulnar artery enter the forearm as it goes between the outspread and radial-ulnar heads. It comprised of two muscle guts isolated by a middle ligament, coordinated proximally to embed itself on to the humerus epicondyle by the shallow leader of the pronator teres muscle.

A2: It arises from the medial epicondyle of the humerus, alongside the other flexor muscles of the forearm. It keeps running on the spiral side of the forearm and lastly joins to the base of the subsequent metacarpal bone and furthermore to the third metacarpal bone by a little slip.

A3: The extensor carpi radialis longus split from its connection on the supracondylar ridge of the humerus into a parallel, intermedial, and medial head. The horizontal and intermedial heads embedded onto its runs of the mill addition site on the subsequent metacarpal base; however, the medial head is embedded onto the extensor carpi radialis brevis muscle.

\subsection{Signal Acquisition and Pre Processing}

Choosing a suitable amount of EMG channels and terminal positions are other significant problems to be measured in signal procurement. Based on the muscle strength and physiological activity the numbers of channel and electrode needles are selected. EMG signals may have a pattern balanced from zero, and normally, the crude sign is amended for standard counterbalancing by deducting the mean amplitude value from the collected EMG signals. The filtering model is characterized as

$F l t(k)=\exp \left(\frac{-p^{2}}{2 p \sigma^{2}}\right)$

EMG signal is changed as a vector and the variety is found. By and large, for the noise evacuation process, a Gaussian sifting is utilized, which is characterized as Flt (k) where "p" represents the frequency and $\sigma$ means a deviation.

\subsection{EMG Signal Features Selection}

The features are excess, and thus joining a portion of the features may not be an outcome in noteworthy development in the accuracy of expectation than utilizing both of them. The worthwhile features of EMG signal are which cause it important for motion to incorporate its straightforwardness in obtaining just as preparing, the advancement of remote and wearable cathodes, and the arrangement of quick and suitable conveying control directions to artificial frameworks. Here two various types of features are extricated from the EMG signals robotic framework. Total Movements considered in our proposed model are six types. The sample limb movements in HAM are depicted in Fig. 1.

M1 Left Rotation; M2 Right Rotation; M3 Close Hand

M4 Open Hand; M5 upper limb; M6 Down limb

\subsubsection{Time Domain Based Features}

Clean types of features are separated straightforwardly from unprocessed EMG signals; these features are effectively distinguished or identified with the motion of the robot system for further prediction procedures. These clean features are important to acquire the distinctions of the featured 
EMG signal. This EMG signal is assessed in time area feature; our proposed model is in excess of ten Time-space features as chosen, and it's clarified in Tab. 1.

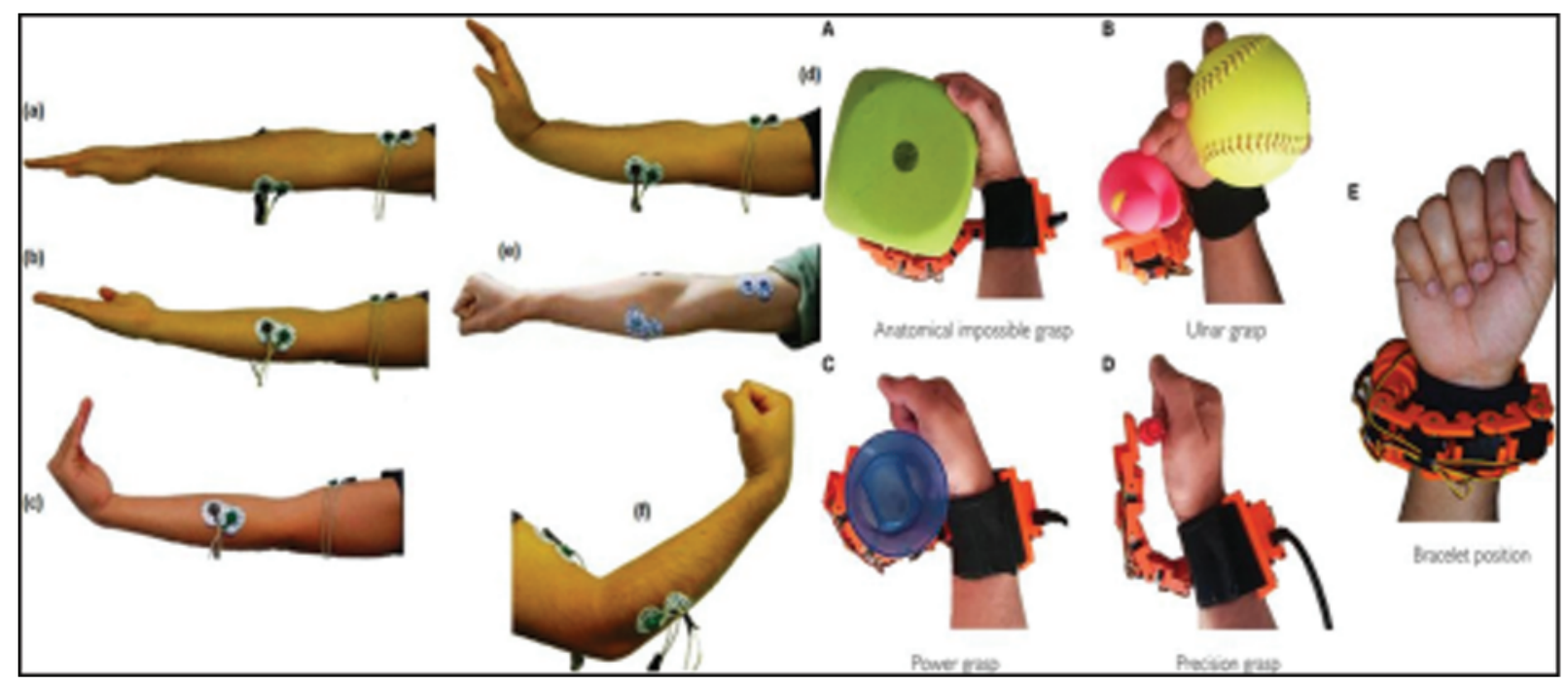

Figure 1: Sample limb movements in HAM

Table 1: Time based features for EMG signal

\begin{tabular}{|c|c|c|}
\hline Feature & Mathematical representation & Description \\
\hline Root mean square (RMS) & $R M S=\sqrt{1 / N} \sum \operatorname{emg}(s)^{2}$ & $\begin{array}{l}\text { The square base of the mean } \\
\text { power of the EMG signal for a } \\
\text { given time frame }\end{array}$ \\
\hline Kurtosis & Kurt $=\frac{E\left(s^{4}\right)}{\left[E(s)^{2}\right]^{2}}-3$ & $\begin{array}{l}\text { A random variable is registered } \\
\text { by separating its fourth } \\
\text { harmonic distortion by the } \\
\text { square value of its scale. }\end{array}$ \\
\hline $\begin{array}{l}\text { Integrated Electromyogram } \\
\text { (IEMG) }\end{array}$ & $I M E G=\sum h(r) e(s-r)+w(s)$ & $\begin{array}{l}\text { These qualities were contrasted } \\
\text { and then compared with relative } \\
\text { paces of oxygen utilization } \\
\text { when the subject performed } \\
\text { equivalent paces of positive and } \\
\text { negative jobs. }\end{array}$ \\
\hline Standard deviation (SD) & $\begin{array}{l}S D= \\
\sqrt{1 / n-1}\left(\sum e m g(s-\vec{s})^{2}\right.\end{array}$ & $\begin{array}{l}\text { It represents noise and other } \\
\text { impedance. It can represent the } \\
\text { signal-to-noise ratio (SNR) of } \\
\text { the signal which can be } \\
\text { controlled by separating the } \\
\text { mean by the standard deviation. }\end{array}$ \\
\hline
\end{tabular}


Table 1: Continued

\begin{tabular}{|c|c|c|}
\hline Feature & Mathematical representation & Description \\
\hline Zero-Crossing (ZC) & $\begin{array}{l}Z C=\sum_{i=1}^{N} \operatorname{sgn}\left(-s_{i}, s_{i+1}\right) \\
\operatorname{sgn}(s)=\left\{\begin{array}{l}1 \text { if } s>0 \\
0 \text { otherwise }\end{array}\right.\end{array}$ & $\begin{array}{l}\text { It tracks the number of } \\
\text { occasions the waveform crosses } \\
\text { zero point, changing from a } \\
\text { positive signal to a negative one, } \\
\text { and the other way around. }\end{array}$ \\
\hline $\begin{array}{l}\text { Slope Sign } \\
\text { Changes (SSC) }\end{array}$ & $S S C=\sum f\left(s_{n}\right)-\left(s_{-1}\right)$ & $\begin{array}{l}\text { It indicates the number of times } \\
\text { slope of the waveform sign } \\
\text { changes in a range of } 20 \mathrm{mV} \text {. }\end{array}$ \\
\hline Waveform Length (WL) & $W L=\sum_{n=1}\left|s_{n+1}, s_{n}\right|$ & $\begin{array}{l}\text { Waveform Length is identified } \\
\text { with waveform amplitude, } \\
\text { frequency and time, combined } \\
\text { length of the waveform in excess } \\
\text { of the time fragment. }\end{array}$ \\
\hline Mean Absolute Value (MAV) & $M A V=1 / p \sum|f(p)|$ & $\begin{array}{l}\text { Time-space variable is estimated } \\
\text { as a component of time. It } \\
\text { symbolizes the area below the } \\
\text { EMG signals which formerly } \\
\text { have been amended; negative } \\
\text { qualities have been made } \\
\text { positive. }\end{array}$ \\
\hline
\end{tabular}

\subsubsection{Time-Frequency Domain-Based Features}

Assessed control range thicknesses that are registered by parametric techniques are Complex Wavelet Transform (CWT) that is being utilized. By applying this change, six arranged wavelet subgroups are created. Among the sub-groups, two Low High (LH) and two High Low (HL) sub-groups are utilized to discover the heading or angle of the surface in a square. These features, for most part, require a change that could be computationally costly. As far as we could possibly know, there is no complete research on extraction of actual signals because of various characteristics of EMG signal demonstration for automated framework execution analysis [23].

\subsubsection{Complex Wavelet Transform (CWT)}

In two-dimensions, CWT is accomplished by applying filters independently along lines and sections. On the off chance that the two channels stifle negative frequencies, the primary quadrant of the range is obtained. CWT is otherwise called complex change since it incorporates the genuine and imaginary piece of six arranged wavelet coefficients.

Block diagram of CWT appears in Fig. 2, where the top tree R produces the genuine pieces of the wavelet coefficient and base tree I creates the nonexistent pieces of the CWT coefficient. $*$ indicates a convolution activity, $\downarrow 2$ implies down inspecting by 2 . Furthermore, there is a low-pass channel and high-pass channel, which structures a Hilbert changed pair to protect the ideal remaking of the discrete 
wavelet transform; [24] With the filter band tree structure, the accompanying wavelet sub-groups are arranged at $\pm 75^{\circ}, \pm 150$ and \pm 450 . Mathematical Expression of this CWT appears as

$$
\begin{aligned}
& \varphi 1(A, B)=\phi(A) \varphi(B) \rightarrow L H \\
& \varphi 2(A, B)=\phi(A) \varphi(B) \rightarrow H L \\
& \varphi 3(A, B)=\phi(A) \varphi(B) \rightarrow H H
\end{aligned}
$$

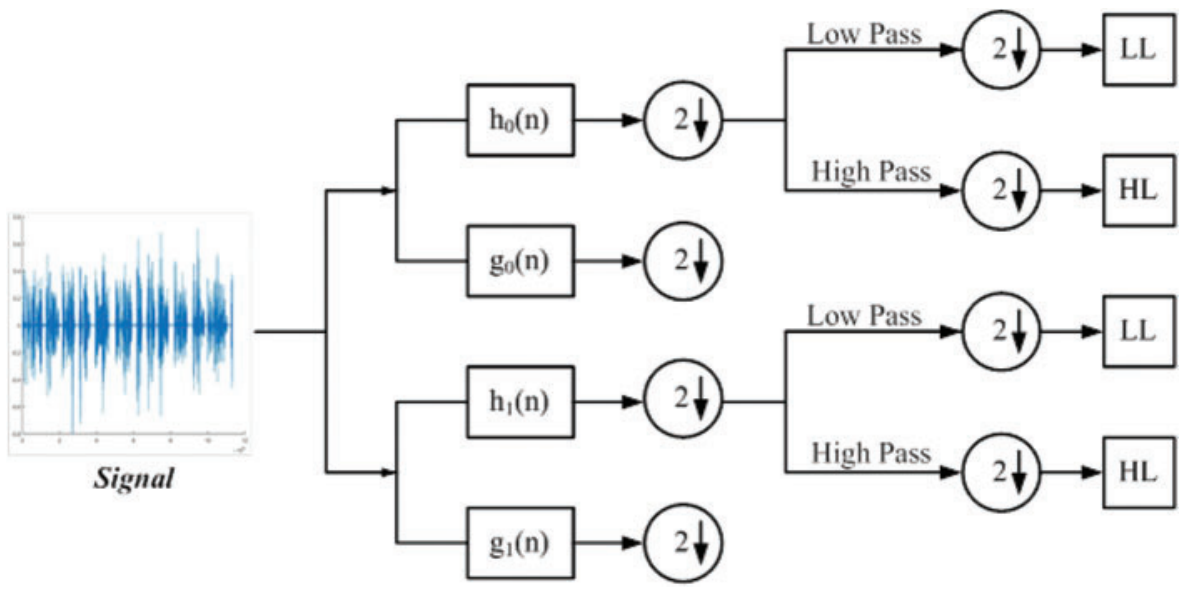

Figure 2: Block diagram of CWT

From the CWT formulations $\varphi(A)$ and $\varphi(B)$ the low pass filter represents alongside the first and second measurements to concentrate the features from the EMG signals. The two filters are situated separately as horizontally and vertically. The HH sub-band is simultaneously oriented along with the $+45^{\circ}$ and $-45^{\circ}$ slant bearings. It is additionally indicated as HHp and HHn. Similarly, two LH subbands and two HL sub-bands are denoted as $L H_{p}, L H_{n} H L_{p}$ and $H L_{n}$ and with these six situated subbands, the best angular mode is evaluated among the 35 angular modes for the intra prediction process.

\subsubsection{Extracted Features for Proposed EMG Signals}

Here absolutely twelve features are extricated from the preprocessed EMG signals; dependent on all features the development of the exoskeleton orthosis is analyzed. Nevertheless, a large portion of the features is repetitive, and consequently joins a portion of the characteristics which does not bring about critical betterment in the precision of forecast than utilizing a signal portion [25]. These features don't just really consider the present worth, and yet can be determined over a window. As the frequency-area includes for the most part, it shows muscle fatigue and variation from the normal due to change in skin temperature.

\subsection{Model-Based Approach for Motor Parameters Prediction}

A linear or nonlinear systematic relationship is built up among information sources and yields the correlation which can think of a few obscure parameters of the data sources to give directions in calculation of the objective yield.

At this point, the parameters are balanced over and over again until the ideal execution of the model is accomplished. In the optimization procedure for the model-based methodology the connection between EMG signals are analyzed in addition to our proposed square graph appearing in Fig. 3. To analyze model-characterized parameters of recorded developments, different programming 
bundles have been created by the MLO model and the method for parameter estimation is analyzed in the next section.

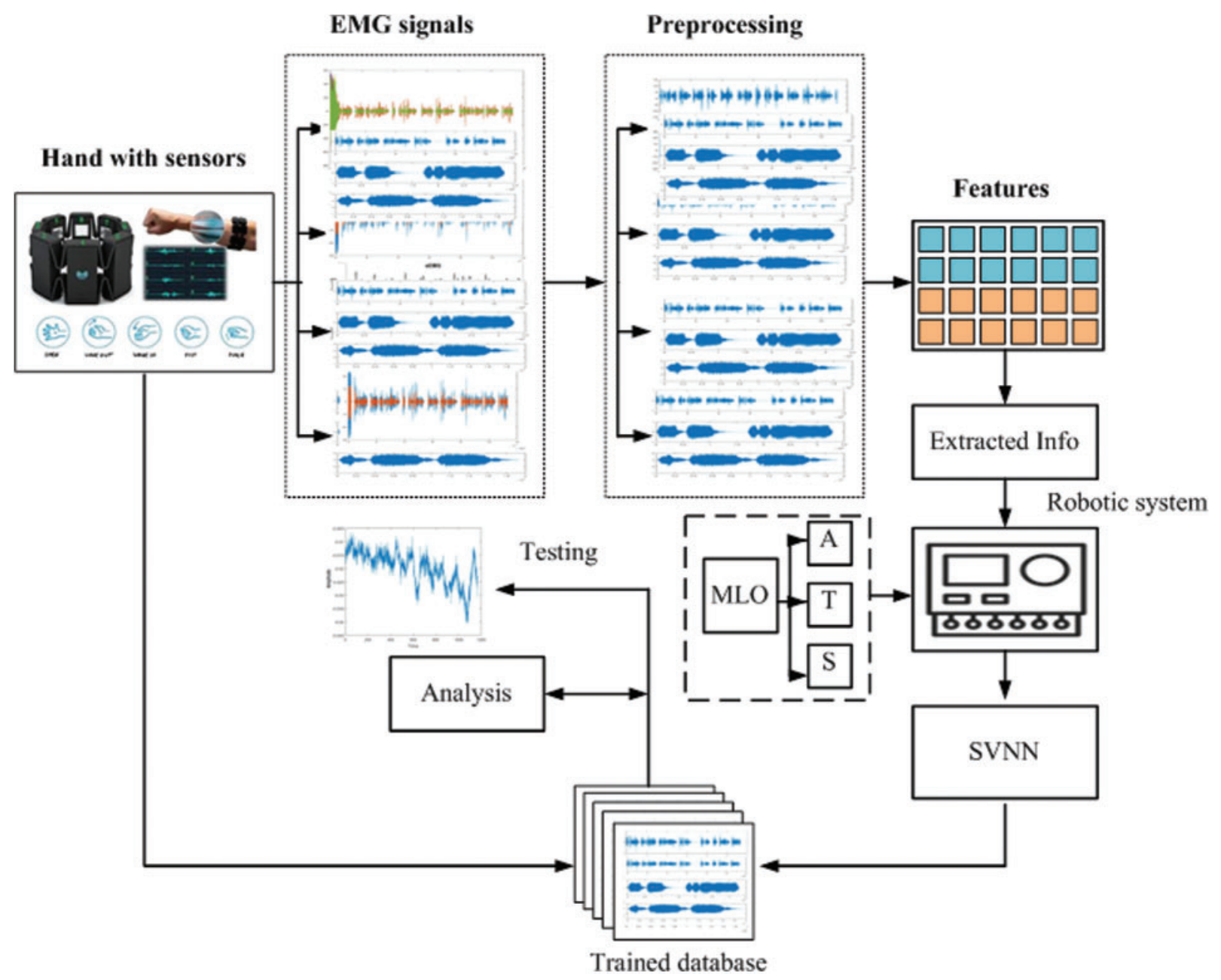

Figure 3: Block diagram for proposed robot collaboration systems

\subsubsection{Adaptive Lion Optimization}

The optimization strategy is part of three sections that are executed on the web while the movements are performed by the subject. Optimization rules are centered on advancing at least one explicit highlight of the development. However, human motor control may be thought about in general assignment attributes as opposed to optimizing at least one of the components.

\subsubsection{Objective Function for Parameter Estimation}

In our proposed EMG investigation different parameters are significant like speed, acceleration and torque. To appraise the torque expectation, we chose not to utilize the exoskeleton joint posture as the contribution of the SVNN in light of the fact that this data is incorporated into the tonic part of the EMG signal. To gauge the torque forecast, we have chosen not to utilize the exoskeleton joint posture as the contribution of the SVNN, on the grounds that this data is incorporated into the tonic segment of 
the EMG signal. In this method, the human robot interaction system just helps the insufficient torque to produce an objective development to upgrade the recuperation of engine elements of patients. In their framework, the robot just helps the insufficient torque to create an objective development to upgrade the recuperation of motor functions of patients.

$T_{E O}=\sum_{i=1}^{n}\left(\left(u_{j}-u\right) * \frac{u_{j}-y_{j}}{u_{j}-y_{j}} * p_{m o t i o n, i}\right)$

In formulation (2) $T_{E O}$ is torque in exoskeleton orthosis, $u_{j}$ is a vector of joint and $y_{j}$ are points of origin and frames, $p_{\text {motion }}$ and is identified by motion estimated in particular movements. Removal is permitted during ordinary activity mode which is however not handled explicitly.

\subsection{The Procedure of MLO Model}

\subsubsection{Hunting Behavior}

These hunters are classified into three subgroups randomly. The group with the most elevated wellness is conceived at the center and the other gathering is conceived as the right and left wings. In the focal point of hunters a dummy prey $S_{\text {prey }}$ is considered and is assessed as follows:

$S_{\text {prey }}=\frac{\sum \text { Hunters or solutions }}{\text { Number of hunters }}$

In the event of hunting, this $S_{\text {prey }}$ is assaulted by the hunters which are chosen randomly. All through hunting, if a hunter builds up its personal fitness, $S_{\text {prey }}$ it can run away from the hunter $\&$ novel position of the prey is,

$S_{\text {prey }}^{\prime}=S_{\text {prey }}+\operatorname{rand}(0,1) \times \% P I \times\left(S_{\text {prey }}-K\right)$

Also, the new position of a center hunter is represented as

$K^{\prime}= \begin{cases}\operatorname{rand}\left(K, S_{\text {prey }}\right), & K<S_{\text {prey }} \\ \operatorname{rand}\left(S_{\text {prey }}, K\right), & K>S_{\text {prey }}\end{cases}$

where, $\operatorname{rand}(\mathrm{x}, \mathrm{y})$ refers to the random esteem among $\mathrm{x}$ and $\mathrm{y} . \mathrm{x}$ and $\mathrm{y}$ represent the lower and upper bound.

\subsubsection{Adaptive Moving Toward a Safe Place}

Rest females of the pride move to a protected domain. As every domain has the best arrangements, the arrangements in ALO can be improved. The new position of the female lion is given in formulation [26].

$M S P_{\mathrm{L}}^{\prime}=M S P_{L}+2 d \times \operatorname{rand}(0,1)\left\{L_{1}\right\}+U(-1,1) \times \tan (\theta) \times d \times\left\{L_{2}\right\}$

$\left\{\mathrm{L}_{1}\right\} \cdot\left\{L_{2}\right\}=0,\left\|\left\{L_{2}\right\}\right\|=1$

where $M S P_{\mathrm{L}}^{\prime}$ represents the female lion position, $\mathrm{d}$ alludes the separation among the FL and the picked position is chosen by tournament selection method from the pride region, $\left\{\mathrm{L}_{1}\right\}$ degree of vector where the beginning point is a previous location of a female lion and its direction is toward the picked point and $\left\{L_{2}\right\}$ is perpendicular to $\left\{\mathrm{L}_{1}\right\}$. 


\subsubsection{Roaming Behavior}

A male lion is transitioning to an area because of certain problems. The wandering nature of male lions causes the lion optimization algorithm to update periodically. The novel situation of nomad lions is produced as pursues,

$S_{i j}=\left\{\begin{array}{l}L_{i j} \text { if } \text { rand }_{j}>\text { prob }_{i} \\ R A N D_{j} \text { otherwise }\end{array}\right.$

From the above formulation, Lij-represents the present situation of nomad lion randjrepresents the uniform random number between $[0,1] \mathrm{RANDj}$-represents the randomly generated vector and probi-represents the probability esteem.

\subsubsection{Migration}

For the migration procedure, some females are picked arbitrarily and are advanced towards the drifters. The new drifter lioness and old vagabond lioness are masterminded by their wellness. By then, the best females among them are chosen arbitrarily and appropriated to pride the position of an unfilled spot of relocated females. This strategy keeps up the conventional assortment of the whole group and offers information among the pride.

\subsubsection{Lions' Population Equilibrium}

In view of the fact that there is reliably balance in lions masses, close to the completion of every cycle, the number of live lions will be restrained. In accordance, the most extraordinary allowable number of sexual directions in drifters as a migrant lion with the optimal wellness esteem is also removed.

\subsubsection{Termination Criteria}

The solutions are refreshed until finding the ideal controller parameters for ordering initiations of various muscles and repetition of data is kept low, limiting the expenses of the optimization autonomous intricacy of the vital motion with time.

\subsection{Controller Parameter Prediction}

In spite of the fact that a solitary detecting methodology is already valuable for objective prediction, it ought to be conceivable to develop prediction accuracy by joining numerous modalities by means of MLO procedure. In each emphasis of the algorithm, the present condition of the body model is coordinated with the condition of the issue as read from the posture sensors. Our Controller prediction processes SVNN classifier model, where the arrangement module comprises a capacity that maps highlight vectors to names. Extracted noise free EMG signal is utilized in prediction of intended motion of the limbs. Fig. 4 demonstrates the forecast model. In particular, it is utilized to appraise upper limb trajectory and the kinematics of appendage motion where the Upper limbs articulation angles are measured. Torque, speed and force required for the motion of the upper limb is predicted. The parameters can be either legitimately or in a roundabout way anticipated from EMG signals [27]. At this point the immediate assessment method is utilized to assess limb parameters and SVNN reads the sensor data and legitimately gives the predicted result. 


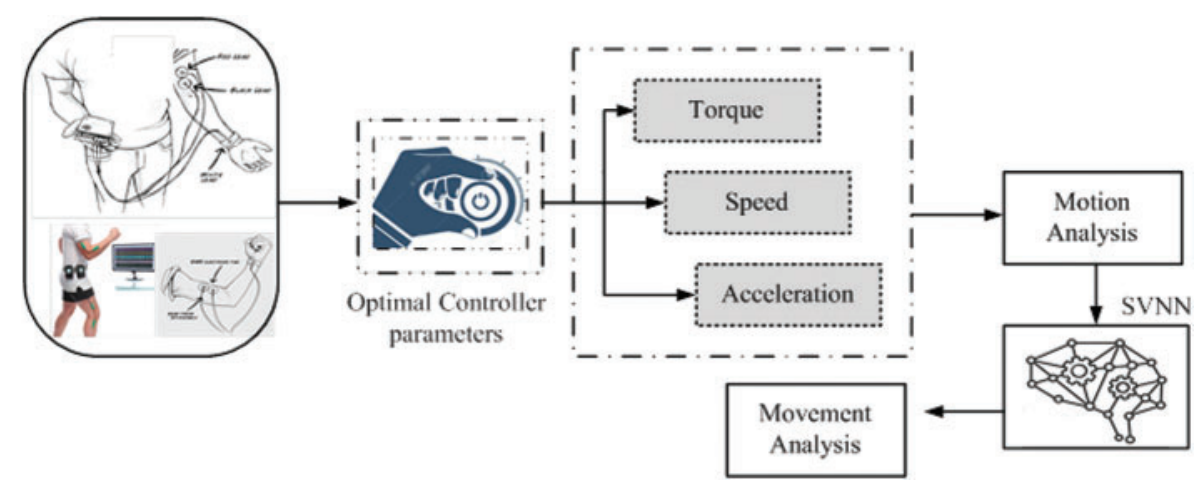

Figure 4: SVNN based controller parameter analysis

\subsubsection{Procedure for $S V N N$}

In the architecture of SVNN, a series of SVNN are masterminded to anticipate the controller parameters to foresee the robotic framework. Considering that the discourse signal has zero amounts of feelings and SVNN units are required by the SVNN classifier, the first Prediction preparing model with the optimal controller parameters is utilized. The yield of SVNN is communicated in formulation.

$S V N N \_$output $_{j}=Q_{1} \times \log \operatorname{sig}\left[\left(\sum_{g=1}^{6} G_{g} * Q_{2}\right)+m_{1}\right]+m_{2}$

Here, the features given to the SVNN classifier are represented as $G_{g}$ and the loads given in the info and hidden layer are represented as $Q_{1}$, and $Q_{2}$ represents the features given to the SVNN classifier that is duplicated with the loads. The biases in the hidden layer and yield layer are represented as $m_{1}$ and $m_{2}$.

\subsubsection{Condition for SVNN}

The capacity characterized for the SVNN is considered as the error work among the ground truth data and the arranged yield. The weighted vectors' Eigenvalues are used to characterize the yield additionally to influence the wellness work. Condition (10) communicates the SVNN's wellness work.

condition $=\varphi_{\max }+\varphi_{\min }+\frac{S}{o} \sum_{j=1}^{o} \mid S V N N-$ output $_{j}-S V N N-$ output $_{j}^{*} \mid$

Here, $S V N N-$ output $_{j}$ and $S V N N-$ output $_{j}^{*}$ represents the output of the SVNN and ground truth data. The regularization factor for yield is denoted by $S . \varphi_{\min }$ and $\varphi_{\max }$ representing the minimum and most extreme Eigen value for the weight vector.

$\varphi=\operatorname{Eigen}\left(K \times K^{U}\right)$

$\varphi_{\max }=\max (\varphi)$

$\varphi_{\min }=\min (\varphi)$ 


\subsubsection{Training the $S V N N$}

The SVNN classifier used in the SVNN is prepared in disengagement with the assistance of the displayed gravitational hunt algorithm as appeared in the Fig. 4. To decide the feelings covered up in the discourse signal, the fitting loads and inclinations have to prepare the SVNN. Deciding the best loads and the predisposition for SVNN, it is considered as the optimization issue. Utilizing the introduced GSA algorithm, the loads and the predisposition engaged with the SVNN are resolved to anticipate the speed, torque, quickening, and a few parameters.

\section{Simulation Results Analysis}

In this section, the performance of the proposed model is investigated, in view of different parameters like accuracy, efficiency, completion time and optimal control parameters alongside the SVNN prediction method. This examination is in excess of 50 EMG signals, which are used with various movements. This model is executed in MATLAB 2018a with i7 processor and 4GB RAM configurations. In addition, this proposed model is compared with other existing predictor and optimization models.

The EMG signal is measured and analyzed to predict the respective torque, speed and force for the HAM motion. The system explains a total of six hand movements and features that are extracted from the EMG signals utilizing complex wavelet transform. Fig. 5 shows the sample EMG signals of limb muscles of a healthy person for analysis [28].

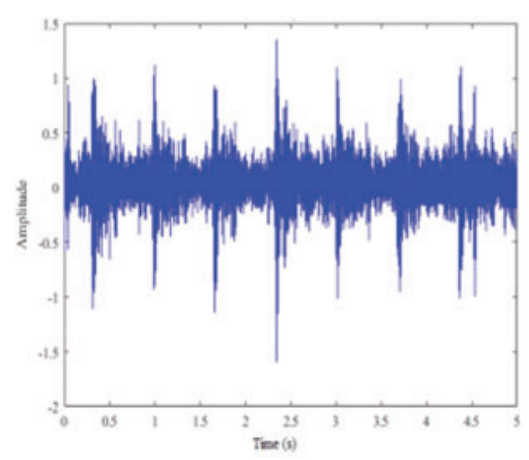

(a)

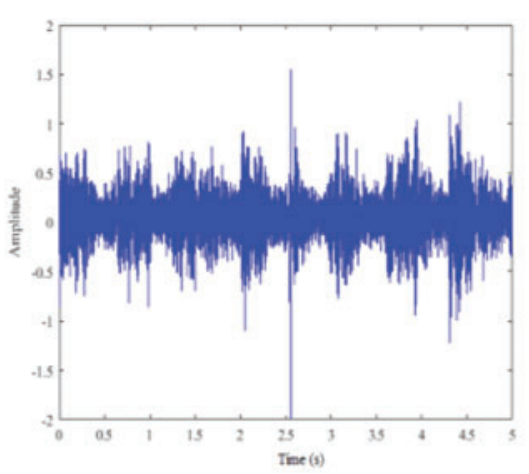

(b)

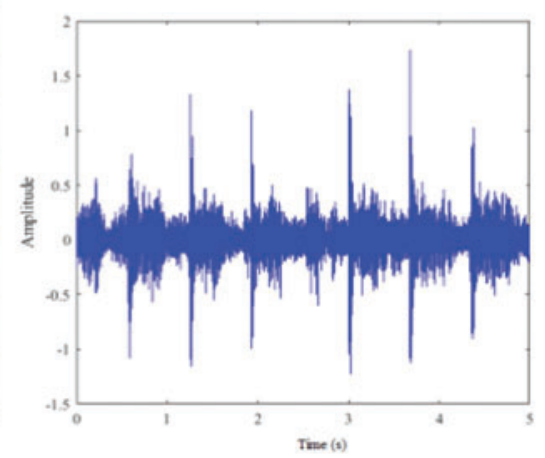

(c)

Figure 5: EMG signals for analysis, (a) Sample-1, (b) Sample-2, and (c) Sample-3

The EMG signals are then handled by utilizing a complex wavelet transform [29]. The complex wavelet transform deteriorates data on time-based frequency. The aftereffects of data handling are done dependent on complex wavelet transform utilizing MATLAB programming. The features extracted are torque, speed and force. It disintegrates the data into eight classifications of data. Figs. 6a-6c speaks to the consequence of wavelet transformations. By utilizing the measured torques, actual joint angle, limb velocity, and acceleration are evaluated. The performance of the features extricated utilizing eight strategies appears in Fig. 7. Noise sensitivity of feature space is depicted through noise resistance. Noise more often than not includes unsettling influence developments with low frequency [30]. Overall, the vitality of wavelet coefficients of EMG signal in eight scales demonstrates a better outcome during averaging when compared with others. Raw wavelet coefficients are impractical to be utilized if extra feature extraction, as well as feature projection, isn't applied. The choice of the heartiest EMG features depends on the consequence of the first test. 


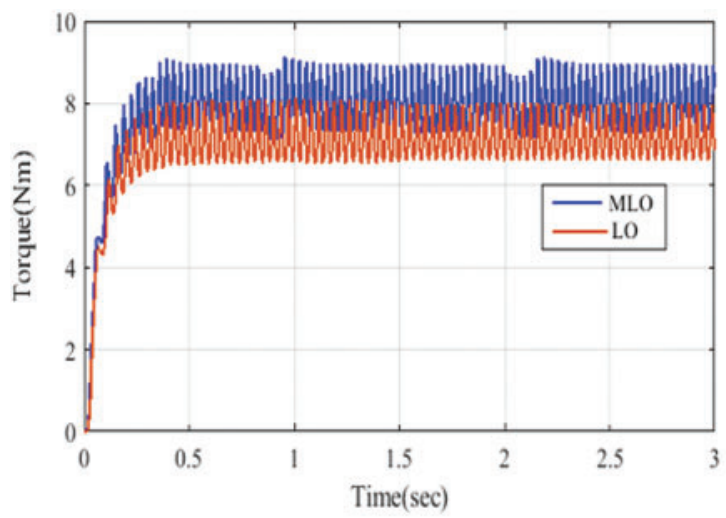

(a)

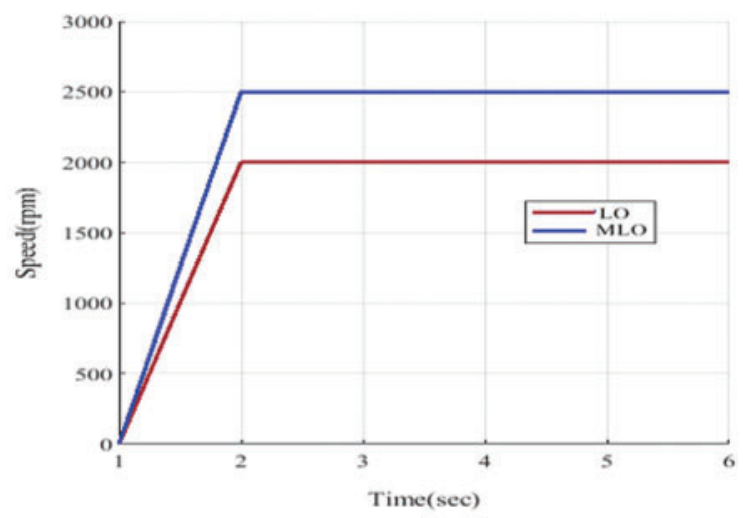

(b)

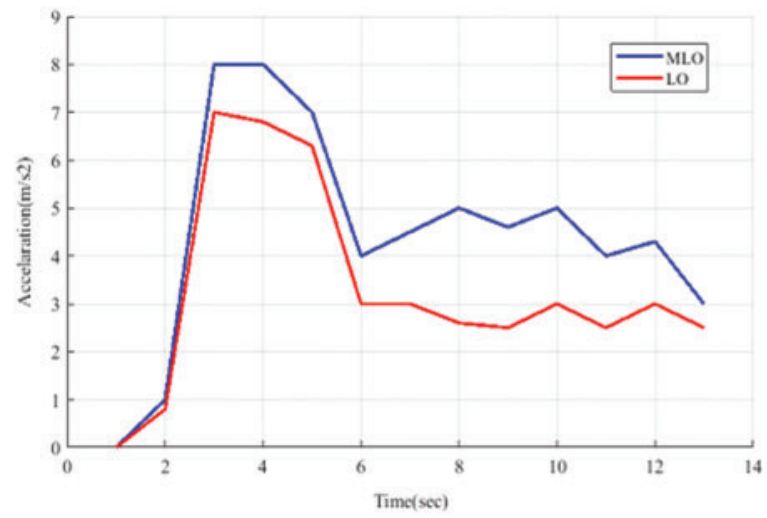

(c)

Figure 6: Optimal parameters analysis of (a) torque, (b) speed and (c) acceleration

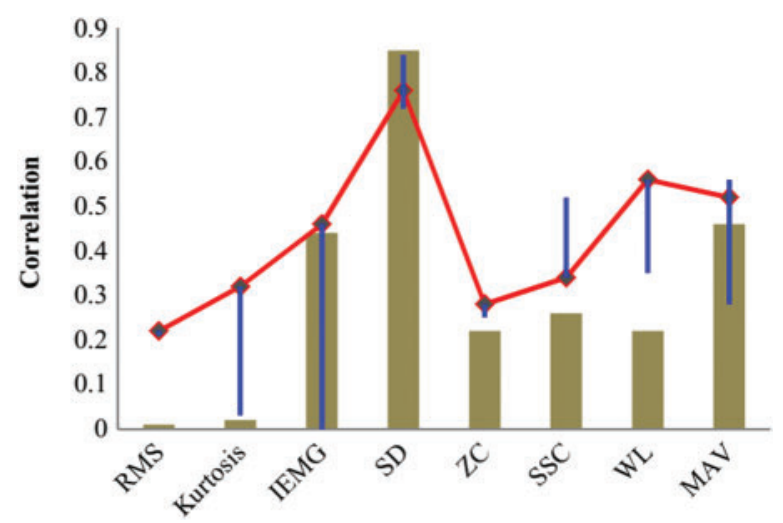

Figure 7: Performance of features

The steady feature ought to prevail with regards to keeping up high classification accuracy. MLO enormously diminishes the component of the optimization however creates a rehashed optimization which is essential when parameters from different muscles are changed. 
Lion optimization is utilized to gain proficiency with the relationship among EMG features from the power of arms. The features are extracted by utilizing a wavelet transform which is referenced in Fig. 8. Moreover, this information is additionally advanced utilizing the Lion Optimization algorithm. The parameters like Torque, Speed, Efficiency, and transmission of data are recorded in Tab. 2. The qualities extricated are throughput, torque, speed, and efficiency. The parameters anticipated from different neural network calculations are recorded in Tab. 3.

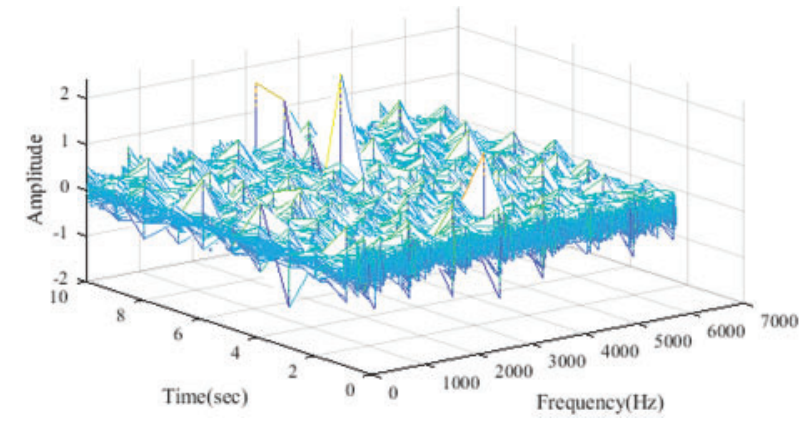

Figure 8: Time-frequency-based features

Table 2: Robotic system, controller parameters analysis

\begin{tabular}{llllll}
\hline Optimization & Signals & $\begin{array}{l}\text { Throughput } \\
(\mathrm{Mbps})\end{array}$ & Torque $(\mathrm{Nm})$ & Speed (rpm) & Efficiency (\%) \\
\hline LO & EMG1 & 62.11 & 1.25 & 1200 & 85.22 \\
& EMG2 & 63.48 & 1.21 & 1300 & 82.14 \\
& EMG3 & 69.22 & 7.51 & 1250 & 76.22 \\
& EMG4 & 76.8 & 7.88 & 1215 & 71.22 \\
MLO & EMG5 & 81.22 & 8.45 & 1800 & 83.22 \\
& EMG1 & 92.22 & 0.25 & 1350 & 92.22 \\
& EMG2 & 93.27 & 7.44 & 1400 & 93.22 \\
& EMG3 & 86.4 & 7.55 & 1500 & 89.84 \\
& EMG4 & 86.9 & 8.59 & 1650 & 87.45 \\
& EMG5 & 85.2 & 8.89 & 1900 & 96.22 \\
\hline
\end{tabular}

The wavelet transform could be basically partitioned into discrete and persistent structures. It productively converts the signals with an adaptable goal in both time-and frequency areas. This characterizes the connection between kind of objective movement and the attributes of estimated EMG information as per the calculation of their applied discriminant function. These estimation results were coordinated with hearing outcome to the subject after pre-exploration of extracting features between consolidated movement and essential movement notwithstanding developed canonical discriminant space. 
Table 3: EMG motion parameter prediction

\begin{tabular}{lllllll}
\hline Technique & M1 & M2 & M3 & M4 & M5 & M6 \\
\hline KNN & 63.22 & 76.22 & 82.22 & 56.22 & 63.22 & 59.22 \\
NN & 65.24 & 81.11 & 81.1 & 53.22 & 69.22 & 69.22 \\
SVM & 69.22 & 79.22 & 76.58 & 82.33 & 68.22 & 65.22 \\
Bayes & 72.22 & 76.85 & 83.52 & 62.22 & 85.22 & 75.22 \\
SVNN & 78.96 & 88.25 & 86.99 & 91.22 & 89.22 & 86.78 \\
\hline
\end{tabular}

Prediction depends on supervised machine learning that takes in prediction rules from recently recorded training data. Throughout training, the classifier Figs. 5-8 out how to orchestrate the patterns. Training results could be altogether diverse, with varieties running from the setting of certain parameters. In Fig. 9a training error is 0.22, its minimum value in epoch 65 and testing error 0.15 in 62 iterations. The minimum error among the feature vectors of the present pattern $\&$ the feature vectors of every training test is determined.

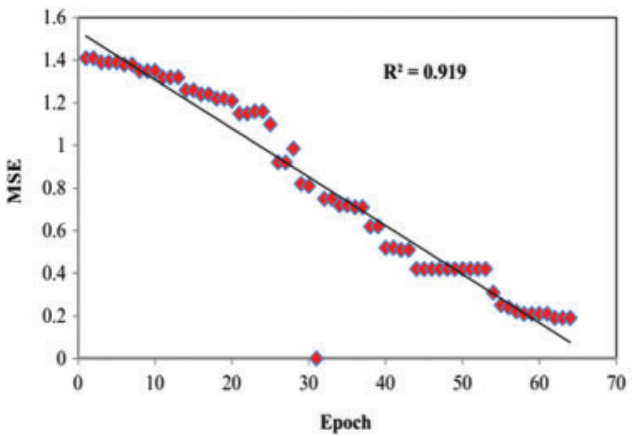

(a)

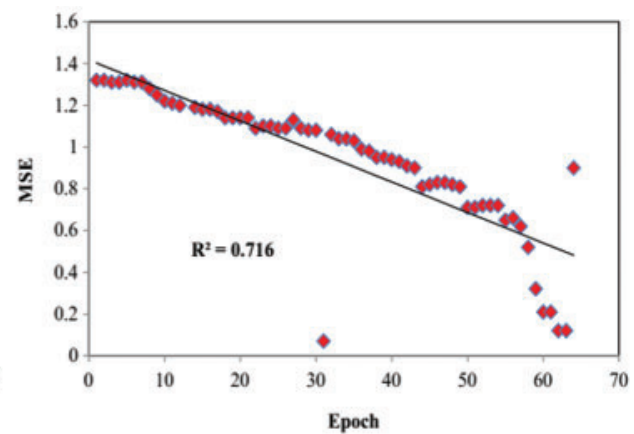

(b)

Figure 9: Prediction SVNN error (a) Training error (b) Testing error

The comparisons of movement prediction accuracy of various algorithms are shown in Fig. 10. This analysis SVM, NN, KNN and Bayes structure are used for comparison in different movements of a limb over various trials. It doesn't have all the assignments of individuals because of a biased classifier as the prediction accuracy throughout rest is somewhat underneath $50 \%$ for 2-object trials. Furthermore, EMG performs ineffectively in subject-free cross-validation and would probably require subject-explicit training. The accuracy of predictions is not the subject to the models and calculations utilized, which are yet in addition to the EMG signal training procedures, as in the required number of channels and characteristics. The accuracy of a proposed model is calculated offline for ideal performance analysis, and such calculated values are kept as the reference values for identification of error measurements. The most extreme accuracy was $96.57 \%$ in M6 different developments. Performance investigation of SVNN-MLO methodologies is analyzed by utilizing the EMG database for various HAM movements dependent on accuracy. The accuracy of the projected classifier is higher than the existing SVM classifier. The request for qualities has been the same for all the filter channels. The proposed MLO-SVNN classifier has performed superiorly to the existing methodology as far as accuracy is concerned with the predictor rate of $85.38 \%$, trailed by the classifier NN and the KNN classifier. The Bayes characterization result without anyone else accomplished an order rate of $96.56 \%$, 
which is the most noticeably awful outcome. In order to sufficiently arrange these developments, more filters ought to be used. Optimal factors, by one limb, can be utilized to gain proficiency with the relationship among EMG features and power created by the contralateral limb in able-bodied subjects, empowering assessment of synchronous and corresponding forces with elevated accuracy.

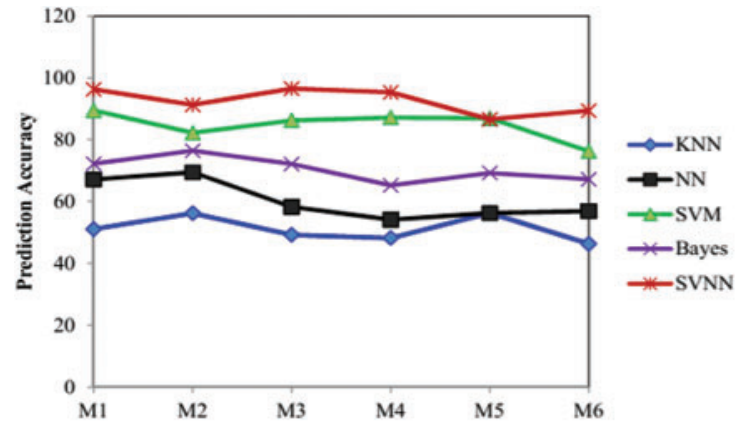

(a)

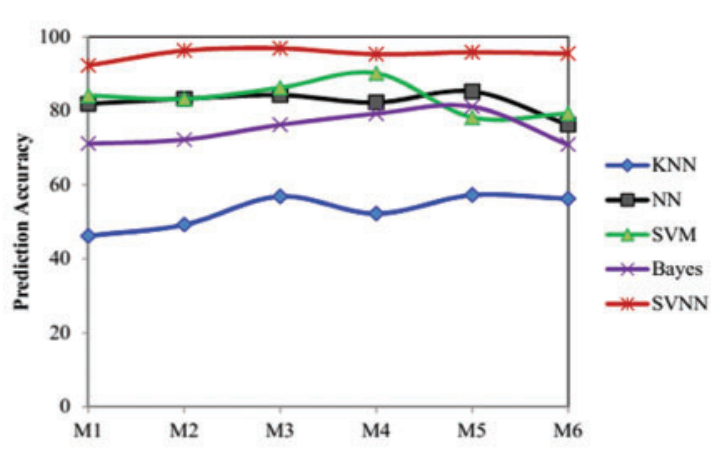

(b)

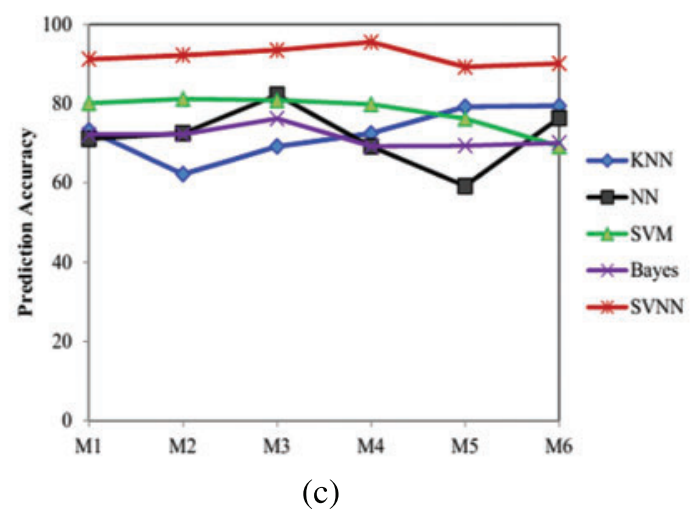

Figure 10: Comparative analysis. (a) Trial 1 (b) Trial 2 (c) Trial 3

\section{Conclusion}

The proposed study presented the Exoskeleton movement predicted by EMG signals. Additionally, it is able to predict the superficially applied forces to the human hands with a hopeful performance via enhanced approaches. The position of the limb is based on the signal generated through the muscle surface which is considered during the data collection process. The EMG signal carries valuable information concerning the hand system. There is an extensive variety of probable purposes using EMG signals in human-machine interfacing. The advantages of MLO and SVNN in EMG prediction resemble a selection operation that keeps better solutions than the worst solutions. The other advantage of the SVM machine learning model is the appropriate kernel function, which can solve any complex problem. The optimal parameters speed, torque, and acceleration are found from the movements of the HAM. Prediction accuracy of SVNN is about $96.56 \%$, compared to other classifiers like SVM, NN, KNN, and bayes. The forces predicted using these two methods were compared with the actual forces of the musculoskeletal demonstrating study as to anticipate the individual muscle forces. Besides, a great deal of research on the estimation of elbow joint torques from EMG signals was also done. In future deep learning and different HAM are planned for analyses. 
In addition, this work is applicable to control predefined HAM movements in robotic systems as demonstrated.

Funding Statement: This work was supported by the Deanship of Scientific Research, King Khalid University, Kingdom of Saudi Arabia under research Grant Number (R.G.P.2/100/41).

Conflicts of Interest: The authors declare that they have no conflicts of interest to report regarding the present study.

\section{References}

[1] M. R. Ahsan, M. I. Ibrahimy and O. O. Khalifa, "Optimization of neural network for efficient EMG signal classification," in 2012 8th Int. Sym. on Mechatronics and Its Applications, Sharjah, United Arab Emirates, pp. 1-6, 2012.

[2] H. Cao, S. Sun and K. Zhang, "Modified EMG-based handgrip force prediction using extreme learning machine," Soft Computing, vol. 21, no. 2, pp. 491-500, 2017.

[3] S. K. Manna and V. N. Dubey, "Comparative study of actuation systems for portable upper limb exoskeletons," Medical Engineering \& Physics, vol. 60, no. 3, pp. 1-13, 2018.

[4] P. K. A. Kostas and J. Kyriakopoulos, "EMG-based position and force estimates in coupled humanrobot systems: Towards emg-controlled exoskeletons," in Experimental Robotics. Vol. 54. Springer, Berlin, Heidelberg: Springer Tracts in Advanced Robotics, pp. 241-250, 2009.

[5] S. J. Chong, S. Al-Ani, C. Pinto and B. Peat, "Bilateral flexor carpi radialis brevis and unilateral flexor carpi ulnaris brevis muscle: Case report," Journal of Hand Surgery, vol. 34, no. 10, pp. 1868-1871, 2009.

[6] B. J. Borbély and P. Szolgay, "Real-time inverse kinematics for the upper limb: A model-based algorithm using segment orientations," BioMedical Engineering OnLine, vol. 16, no. 1, pp. 21, 2017.

[7] Y. A. Meslemi, N. Anwer and L. Mathieu, "Modeling key characteristics in the value chain of additive manufacturing," Procedia CIRP, vol. 70, no. 1, pp. 90-95, 2018.

[8] C. Fleischer and G. Hommel, "Embedded control system for a powered leg exoskeleton," in: Embedded Systems —Modeling, Technology, and Applications. Dordrecht: Springer, pp. 177-185, 2006.

[9] C. Fleischer, C. Reinicke and G. Hommel, "Predicting the intended motion with EMG signals for an exoskeleton orthosis controller," in 2005 IEEE/RSJ Int. Conf. on Intelligent Robots and Systems, Edmonton, AB, Canada, pp. 2029-2034, 2005.

[10] M. Millard, M. Sreenivasa and K. Mombaur, "Predicting the motions and forces of wearable robotic systems using optimal control," Frontiers in Robotics and AI, vol. 4, pp. 456, 2017.

[11] D. Novak, X. Omlin, R. L. Hess and R. Riener, "Predicting targets of human reaching motions using different sensing technologies," IEEE Transactions on Biomedical Engineering, vol. 60, no. 9, pp. 26452654, 2013.

[12] M. Arozi, F. T. Putri, M. Ariyanto, K. A. M. Munadi and J. D. Setiawan, "Electromyography (EMG) signal recognition using combined discrete wavelet transform based adaptive neuro-fuzzy inference systems (ANFIS)," AIP Conference Proceedings, vol. 1788, no. 1, pp. 030116, 2016.

[13] R. Boostani and M. H. Moradi, "Evaluation of the forearm EMG signal features for the control of a prosthetic hand," Physiological Measurement, vol. 24, no. 2, pp. 309-319, 2003.

[14] C. Fleischer, K. Kondak, C. Reinicke and G. Hommel, "Online calibration of the EMG to force relationship," in 2004 IEEE/RSJ Int. Conf. on Intelligent Robots and Systems (IROS), Sendai, Japan, vol. 2, pp. 1305-1310, 2004.

[15] Z. Zhang, K. Yang, J. Qian and L. Zhan, "Real-time surface EMG pattern recognition for hand gestures based on an artificial neural network," Sensors, vol. 19, no. 14, pp. 3170, 2019.

[16] J. L. G. Nielsen, S. Holmgaard, N. Jiang, K. B. Englehart, D. Farina et al., "Simultaneous and proportional force estimation for multifunction myoelectric prostheses using mirrored bilateral training," IEEE Transactions on Biomedical Engineering, vol. 58, no. 3, pp. 681-688, 2011. 
[17] M. Simão, P. Neto and O. Gibaru, "EMG-based online classification of gestures with recurrent neural networks," Pattern Recognition Letters, vol. 128, no. 2, pp. 45-51, 2019.

[18] A. K. Mukhopadhyay and S. Samui, "An experimental study on upper limb position invariant EMG signal classification based on deep neural network," Biomedical Signal Processing and Control, vol. 55, no. 1, pp. $101669,2020$.

[19] M. E. Benalcazar, C. E. Anchundia, J. A. Zea, P. Zambrano, A. G. Jaramillo et al., "Real-time hand gesture recognition based on artificial feed-forward neural networks and emg," in 2018 26th European Signal Processing Conf. (EUSIPCO), Rome, pp. 1492-1496, 2018.

[20] N. Duan, L. Z. Liu, X. J. Yu, Q. Li and S. C. Yeh, "Classification of multichannel surface-electromyography signals based on convolutional neural networks," Journal of Industrial Information Integration, vol. 15, no. 15, pp. 201-206, 2019.

[21] M. H. Quazi and S. G. Kahalekar, "Artifacts removal from EEG signal: FLM optimization-based learning algorithm for neural network-enhanced adaptive filtering," Biocybernetics and Biomedical Engineering, vol. 37, no. 3, pp. 401-411, 2017.

[22] X. Fang, D. Pang, J. Xi and X. Le, "Distributed optimization for the multi-robot system using a neurodynamic approach," Neurocomputing, vol. 367, no. 12, pp. 103-113, 2019.

[23] S. Güneş, M. Dursun, K. Polat and Ş. Yosunkaya, "Sleep spindles recognition system based on time and frequency domain features," Expert Systems with Applications, vol. 38, no. 3, pp. 2455-2461, 2011.

[24] X. Xie, P. Carré, C. Perrine, Y. Pousset, N. Zhou et al., "Reduced-reference image quality metric based on statistic model in complex wavelet transform domain," Signal Processing: Image Communication, vol. 74, pp. 218-230, 2019.

[25] P. Qin and X. Shi, "Evaluation of feature extraction and classification for lower limb motion based on sEMG signal," Entropy, vol. 22, no. 8, pp. 852, 2020.

[26] M. Yazdani and F. Jolai, "Lion optimization algorithm (LOA): A nature-inspired metaheuristic algorithm," Journal of Computational Design and Engineering, vol. 3, no. 1, pp. 24-36, 2016.

[27] N. A. Almansour, H. F. Syed, N. R. Khayat, R. K. Altheeb, R. EmadJuri et al., "Neural network and support vector machine for the prediction of chronic kidney disease: A comparative study," Computers in Biology and Medicine, vol. 109, no. 12, pp. 101-111, 2019.

[28] J. Pinchin, G. Smith, C. Hill, M. Chris and I. Loram, "The potential of electromyography to aid personal navigation," in Proc. of the 27th Int. Technical Meeting of the Satellite Division of The Institute of Navigation, Tampa, Florida, pp. 1609-1615, 2014.

[29] J. Chen, S. Bi, G. Zhang and G. Cao, "High-density surface EMG-based gesture recognition using a 3d convolutional neural network," Sensors, vol. 20, no. 4, pp. 1201, 2020.

[30] J. P. A. Joel, R. J. S. Raj, C. A. D. Durai and R. Vedaiyan, "Human adaptive mechatronics system integrated with cybernetics loop using neuromuscular controller in occupational therapy for elderly person with disability," Journal of Ambient Intelligence and Humanized Computing, vol. 12, no. 7, pp. 7299-7308, 2021. 